\title{
Application of Swingler's Method for Analysis of Multicomponent Exponentials with Special Attention to Non-equispaced Data
}

\author{
Rahat Hasan \\ School of Engineering \\ University of Waikato, Hamilton, New Zealand \\ rh163@students.waikato.ac.nz
}

\author{
Jonathan Scott \\ School of Engineering \\ University of Waikato, Hamilton, New Zealand
}

\begin{abstract}
Swingler enhanced the work of Gardner to provide an elegant deconvolution method by which multiple summed exponential components might be resolved within time-domain data. Nevertheless, the application of the method remains limited owing to subtle complications that discourage many users. We present a tutorial and extend the approach to handle nonequispaced data. Finally the method's limits are identified in the case of closely-spaced exponential components with added input noise.
\end{abstract}

\section{INTRODUCTION}

Several authors have employed deconvolution to extract multicomponent exponential components from a transient curve [1]-[5]. The preferred method is attributed to Swingler [2] whose work built upon the original article by Gardner [1]. The method notes that for a time-domain function of the form

$$
f(t)=A_{1} e^{-\alpha_{1} t}+A_{2} e^{-\alpha_{2} t}
$$

where $A_{1}$ and $A_{2}$ are the magnitudes of the two exponential components and $\alpha_{1}$ and $\alpha_{2}$ are the reciprocals of the time constants, delta functions exist such that

$$
A_{1} \delta\left(x-p_{1}\right)+A_{2} \delta\left(x-p_{2}\right)=\mathcal{F}^{-1} \frac{\mathcal{F}\left[\frac{\delta f\left(e^{x}\right)}{\delta x}\right]}{\mathcal{F}\left[-e^{x} e^{\left(-e^{x}\right)}\right]}
$$

where $x=\ln (t)$ and $p=-\ln (\alpha)$. The method generalises to $n$ exponentials straightforwardly. In other words, a multiexponential function can in principle be operated upon to yield a series of delta functions each of whose amplitude and delay indicate the amplitude and decay constant of the corresponding exponential that was summed into the initial function. The algorithm consists of moving to a log-time scale, differentiating, and deconvolving the response signal $-e^{x} e^{\left(-e^{x}\right)}$ in the "trans-log" domain.

Data obtained from simulators and measurement instruments may be non-equispaced in nature, and in any case the move to log-time is highly nonlinear, with the outcome that real-world data presents to the Fourier transform at the start of the deconvolution process in a form that is not only nonequispaced, but potentially very difficult to interpolate. None of the literature addresses this. In [2], computed data is used and no mention is made of the interpolation issue with the Fourier Transform (FT) that is used. In this manuscript we

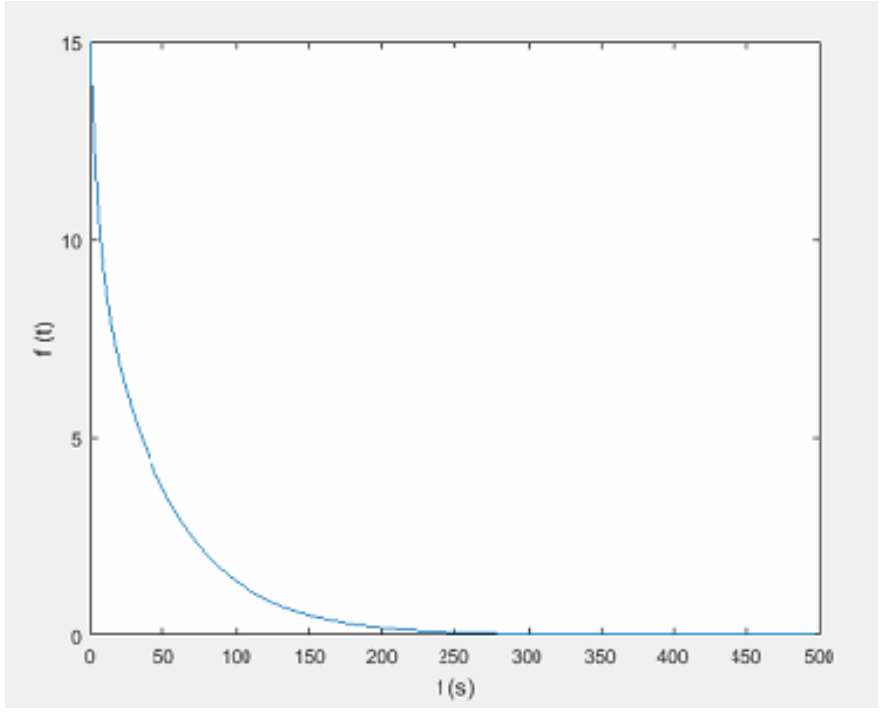

Fig. 1: Transient curve with two exponential functions

describe the series of intermediate steps applied to the nonequispaced data prior to deconvolution, and unlike Swingler we find that we do not need to window data artificially.

\section{STEP BY STEP PROCEDURE}

A transient curve with two exponential functions, $f(t)=$ $5 e^{-0.2 t}+10 e^{-0.02 t}$ is shown in figure 1 by way of example. The first step is to interpolate within the first three data points in order to add more points to the negative axis in the log-time domain. This interpolation is linear in the time domain, since $e^{x} \approx x$ for small $x$, giving an exponential. The transformation of the transient curve to the log-time domain is shown in figure 2 .

Once the transient curve has been transformed to the logtime domain, the transformed curve is then taken to an equally spaced grid using cubic spline interpolation, since most FFT algorithms only accept equispaced data. We have investigated the possibility of transforming directly from the non-equispaced data in the log-time domain to equispaced data in the trans-log domain using the algorithm from [6]. In this case, where the data is comfortably oversampled, the results are identical. Nevertheless, this approach could offer advantage 


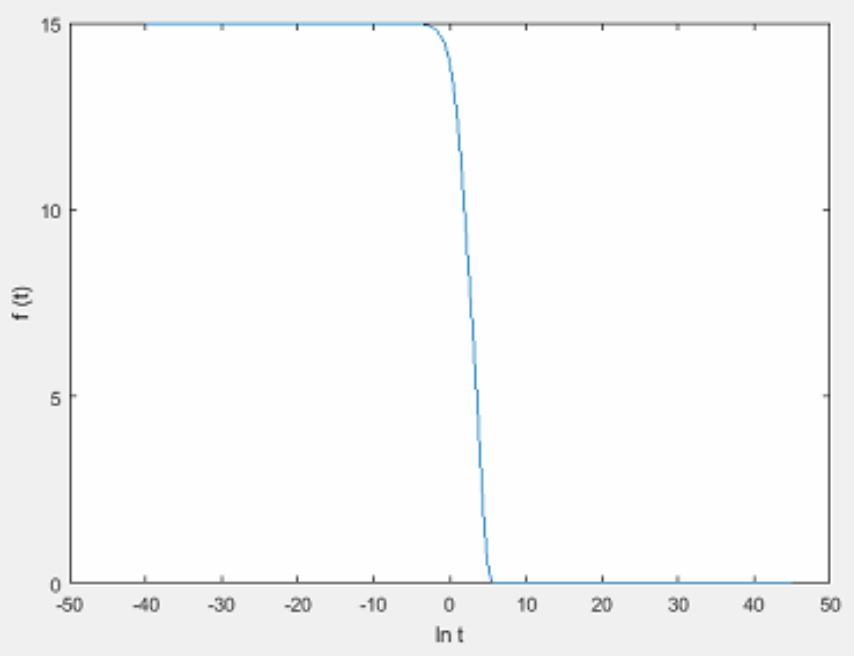

Fig. 2: Transient curve in log-time domain

where the data is sparse. Once on a regular grid in log-time domain, a first-order difference is taken. The resulting curve is shown in figure 3. Looking at this curve in our example, it is possible to see what we know be two blurred peaks, as we chose two functions of comparable magnitude sufficiently far apart. Of course, the peaks will not always be visible in the first-order derivative curve.

The first-order derivative curve is then deconvolved with the response function, $g(x)=-e^{x} e^{\left(-e^{x}\right)}$. This response function is shown in figure 4 . The deconvolution is a point-wise division in the frequency domain. This process is acknowledged to be very sensitive to noise. In our case we can calculate the denominator to any desired precision. To improve the clarity of the outcome it is a well-known technique to add a small constant to the response function in the Fourier domain as required [3]. Figure 5 shows two delta functions with time constants of 6.5 seconds (30\% error) and 59 seconds (18\%

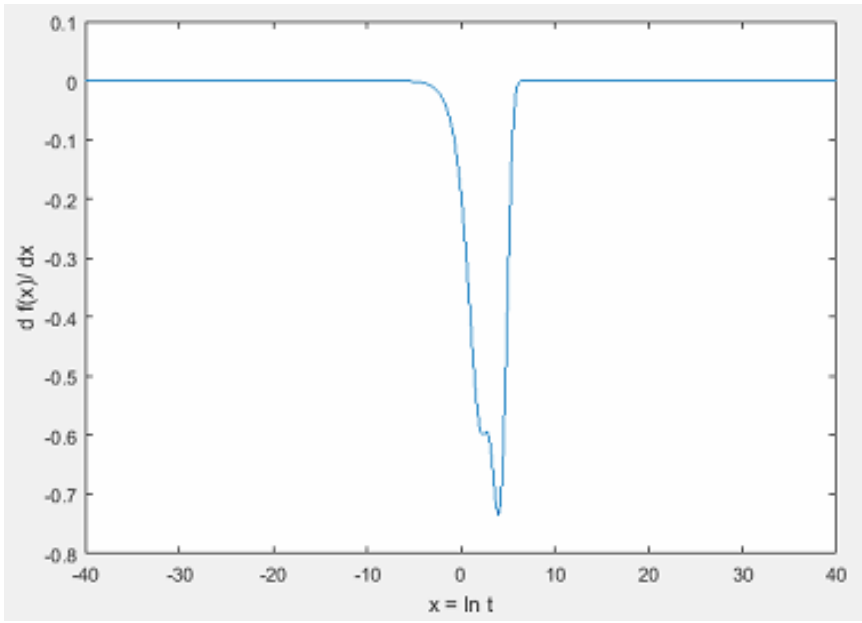

Fig. 3: First-order derivative of the transient curve in log-time domain

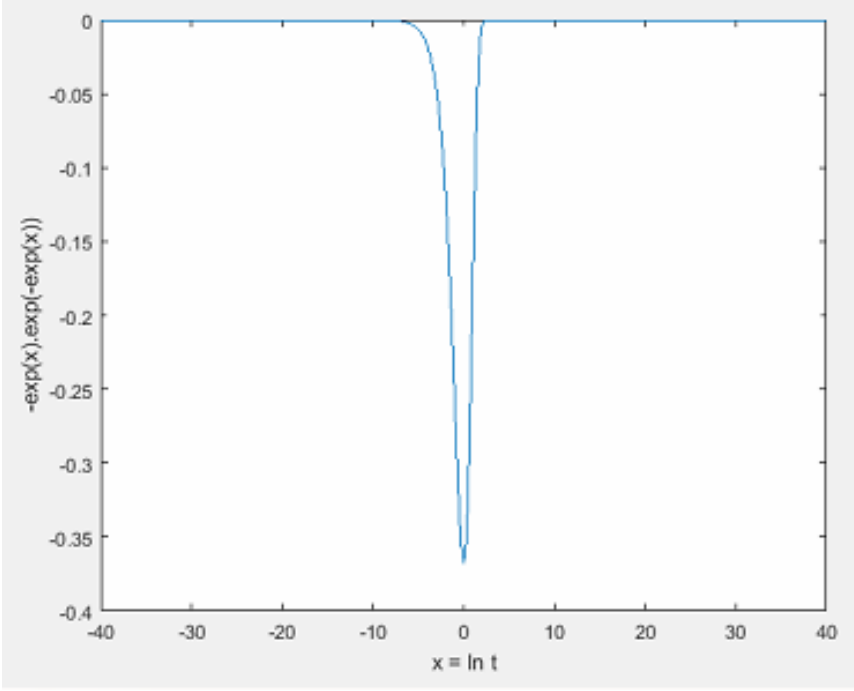

Fig. 4: Plot of the convolving 'response curve'

error) respectively.

\section{VARIATION OF THE NOISE FLOOR WITH RESPECT TO $X$-AXIS RANGE}

In this section, we will address the sensitivity of the delta functions with respect to the variation of the $\mathrm{x}$-axis range. The transient and the response functions used in our algorithm will eventually reach zero as $\mathrm{x}$ approaches infinity but we are free to choose the $\mathrm{x}$-axis range. The operating range for the $\mathrm{x}$ axis values can be determined empirically. Figure 6 shows the impact of changing that range. For an $\mathrm{x}$-axis range of -30 to 30 , the noise floor drops to double precision numerical noise. Further reduction of the range shifts up the noise floor above $-270 \mathrm{~dB}$ through rectangular windowing.

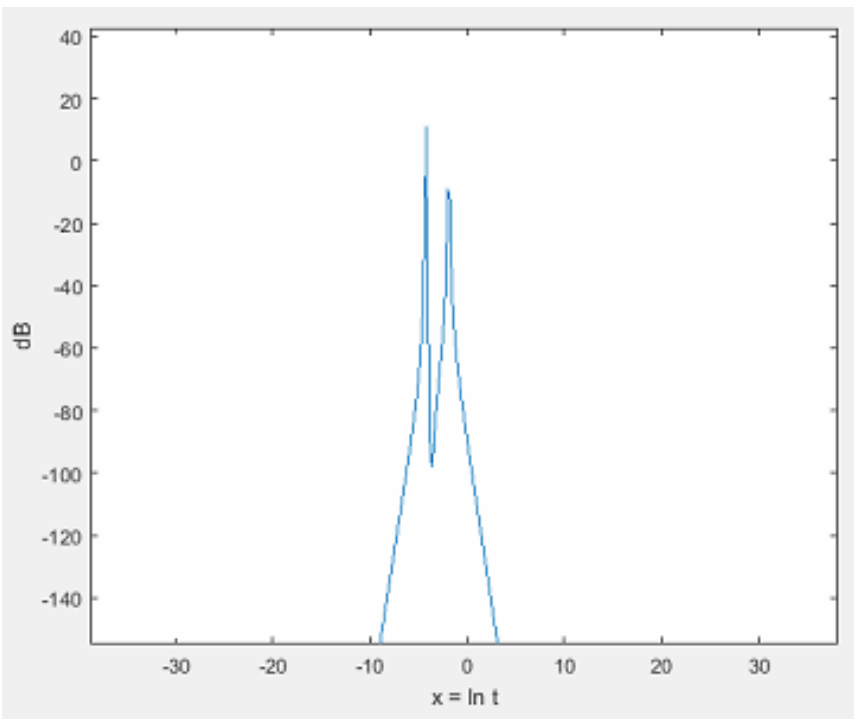

Fig. 5: The resulting curve containing two delta functions 


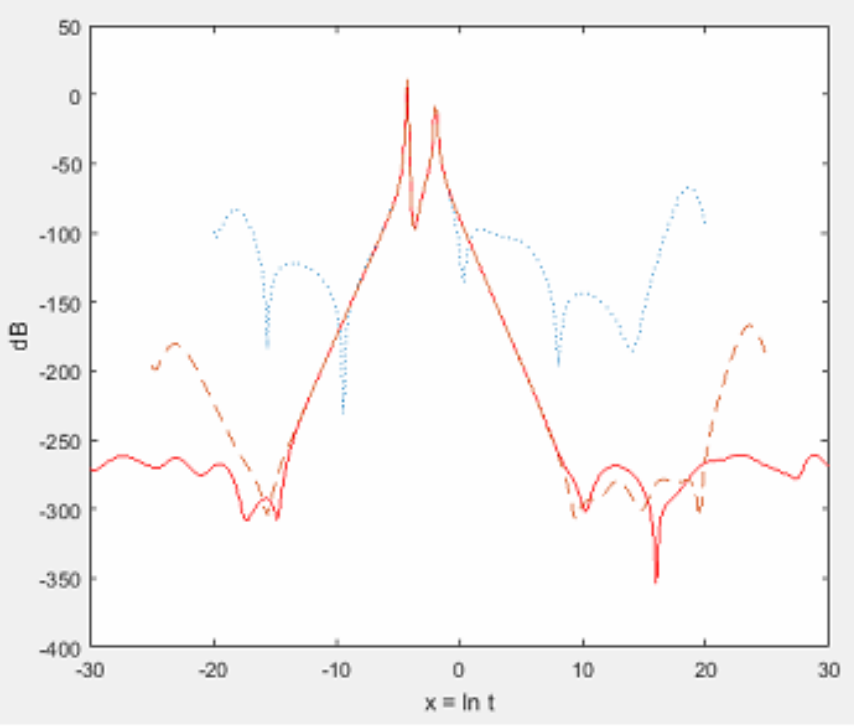

Fig. 6: Variation of the noise floor with respect to $\mathrm{x}$-axis range

\section{COMPARISON USING SWINGLER'S EXAMPLE}

A similar investigation is carried out with the signal used in [2]. The transient function, $f(t)=$ $12.6 e^{-0.1433 t}+2.2 e^{-0.0055 t}+0.9 e^{-0.00042 t}+13.3 e^{-0.000042 t}$ has four exponential functions and for the purpose of this article, the signal is generated with non-equispaced data. The function is deconvolved with the known wrapped around response function, $g(x)=-e^{x} e^{\left(-e^{x}\right)}$ and since the numerical noise in the data is minute, a small noise reduction constant of $10^{-10}$ is added to improve the clarity of the peaks. The noise reduction constant will increase significantly will the addition of artificial noise to the data. The resulting curve is shown below in figure 7 . The time constants of the exponential functions are calculated from the $\mathrm{x}$-axis and compared with the results obtained in [2].

\begin{tabular}{|l|l|l|}
\hline \multicolumn{3}{|c|}{ Time constant values for exponential components } \\
\hline Actual value & $\begin{array}{l}\text { Modified algorithm } \\
\text { (\% error) }\end{array}$ & $\begin{array}{l}\text { Swingler's algorithm } \\
\text { (\% error) }\end{array}$ \\
\hline 6.98 & $9.03(29 \%)$ & $11.02(58 \%)$ \\
181.81 & $221.41(22 \%)$ & $270.42(49 \%)$ \\
$2,380.95$ & $2,980.96(25 \%)$ & $4,447.07(87 \%)$ \\
$23,809.52$ & $26,903.19(13 \%)$ & $32859.62(38 \%)$ \\
\hline
\end{tabular}

TABLE I: Comparison of the values obtained from the delta functions using modified and original algorithms.

From table I, observe that our implementation of Swingler's method achieves superior results compared with the original. The amplitudes of the delta functions are less degraded.

\section{DISCRIMINATING ADJACENT PEAKS}

We now address the issue of the limitation when discriminating adjacent peaks. To investigate this, we examine a

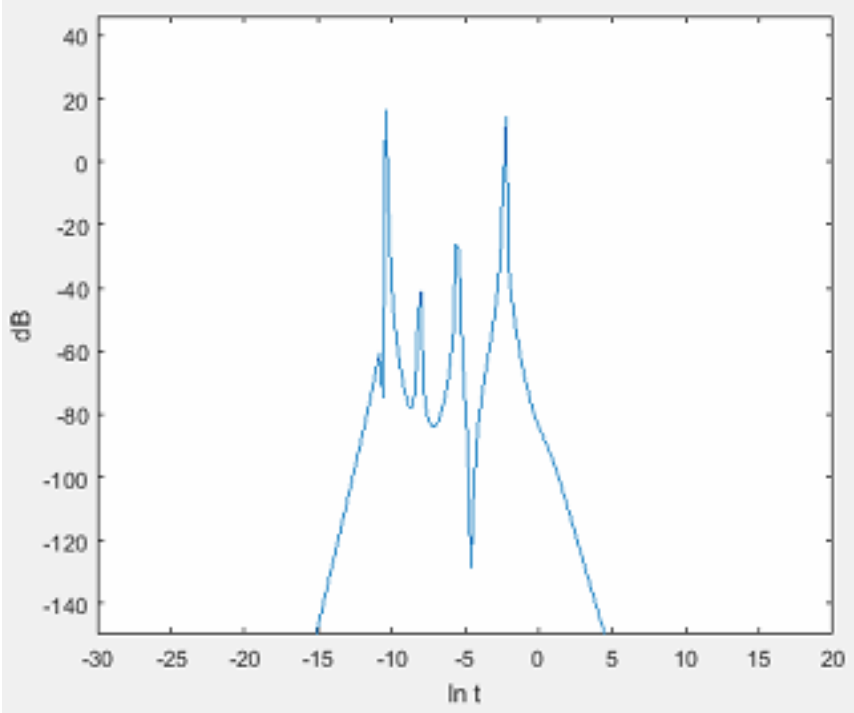

Fig. 7: The resulting curve containing four delta functions

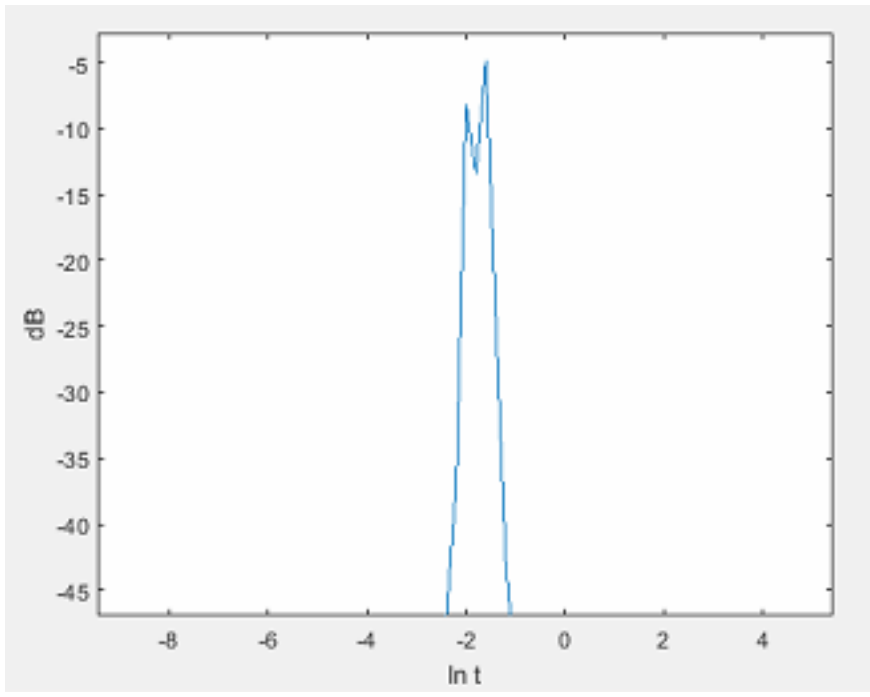

Fig. 8: Two exponentials with time constant ratio of 0.70

number of non-equispaced transient curves with two, closelyspaced exponential functions.

Figure 8 shows the output of the algorithm operating on $f(t)=5.4 e^{-0.28 t}+5 e^{-0.2 t}$ where the ratio of the time constants is 0.7 . When the ratio approaches 1 by even a small amount, the adjacent peaks merge together; figure 9 shows the case for a ratio of 0.74 . Note that the absolute value of the time constants makes no difference, it is only their ratio that determines proximity.

\section{NOISE ANALYSIS}

Real data contains noise. As an example, $-90 \mathrm{~dB}$ additive white Gaussian noise (AWGN) is applied to the function used in Section IV. One of the fundamental limitations of this technique is that without the addition of any constant, the 


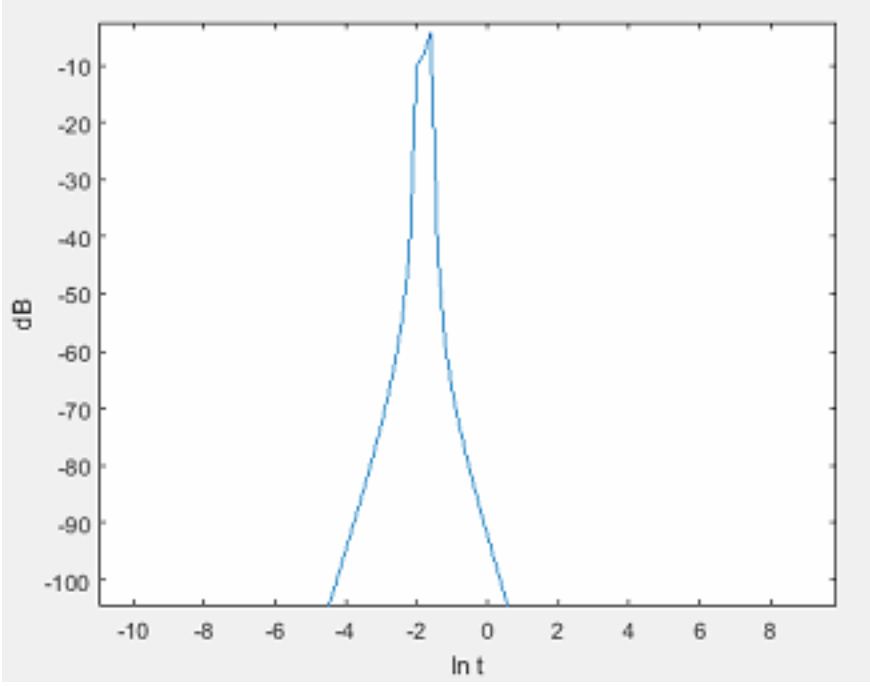

Fig. 9: Two exponentials with time constant ratio of 0.74

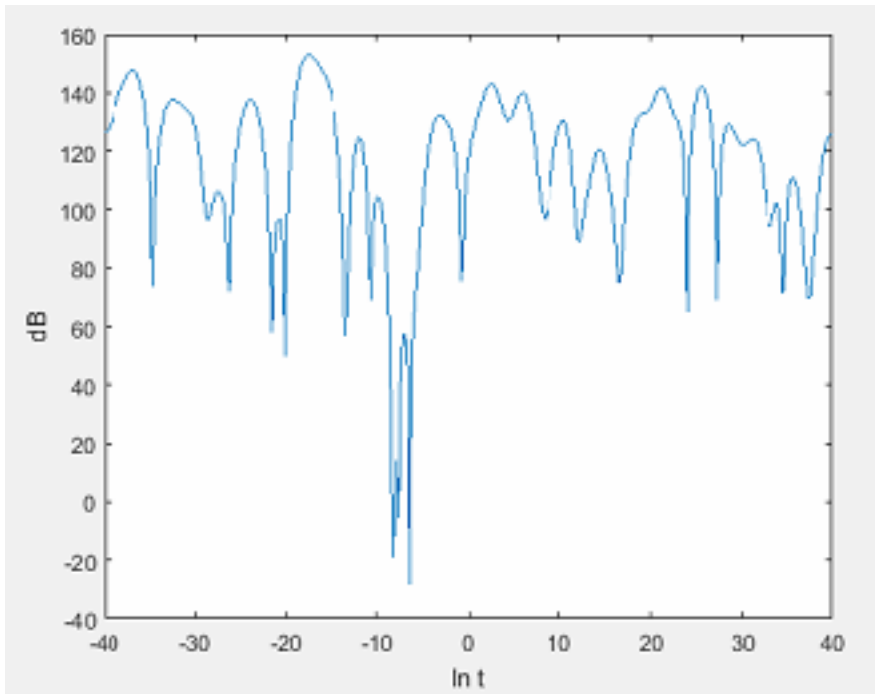

Fig. 10: Presence of noise flooding out the peaks

noise will flood out the peaks of the delta functions as shown in figure 10 .

Trial and error quickly shows that a noise reduction parameter of 0.01 optimally cleanses the peaks. Figure 11 shows the result. The peak values remain unaffected compared to the noise-free case (Section IV). Nevertheless, additional scalloping attends each peak. This is to be expected, as the noise reduction parameter effectively reduces the amplitude of the deconvolution division going outwards from the centre region; this is equivalent to adding a window whose effect after the IFFT is to introduce a series of lesser peaks. This is one of the other fundamental limitations of this technique. The results obtained in this case hold for both equispaced and non-equispaced data.

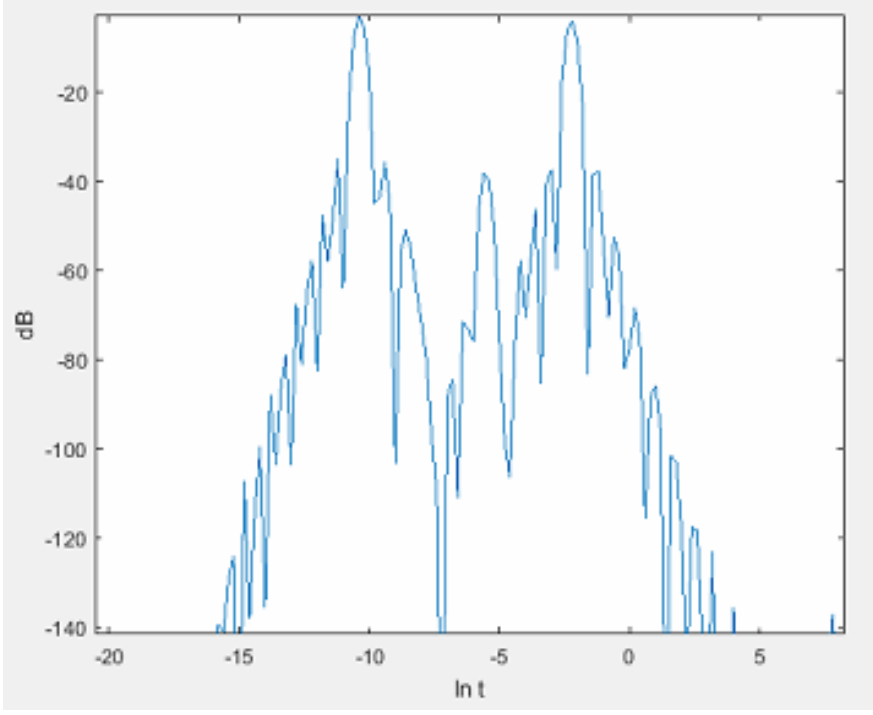

Fig. 11: Addition of a constant making the peaks to stand out from the noise

\section{CONCLUSION}

Through smart interpolation and careful $\mathrm{x}$-axis range selection, we have demonstrated that the method of [2] can straightforwardly produce better approximations of time constant values without the need of windowing, and can handle non-equispaced data. A limitation of this technique is its ability to discriminate delta functions and we quantify this limit. It is also noted that this technique requires a carefullychosen noise reduction parameter to deal with noisy data.

\section{ACKNOWLEDGMENT}

The authors would like to thank Dr Marcus Wilson for helpful discussions.

\section{REFERENCES}

[1] D. G. Gardner, J.C. Gardner and W.W. Meinke, "Method for the Analysis of Multicomponent Exponential Decay Curves", THE JOURNAL OF CHEMICAL PHYSICS, Vol. 31, No. 4, 1959, pp. 978-986.

[2] D. N. Swingler, "A Differential Technique for the Fourier Transform Processing of Multicomponent Exponential Functions", IEEE Transactions on Biomedical Engineering, vol. BME-24(4), 1977, pp408-410.

[3] Abdussamad U. Jibia, and Momoh-Jimoh E. Salami, "Analysis of transient multiexponential signals using exponential compensation deconvolution", Measurement, vol. 45, 2012, pp. 19-29.

[4] Abdussamad U. Jibia, Momoh-Jimoh E. Salami, Othman O. Khalifa, and A.M. Aibinu, "Analysis of transient multiexponential signals using cepstral deconvolution", Applied and Computational Harmonic Analysis, vol. 29, 2010, pp. 88-96.

[5] Abdussamad U. Jibia, and Momoh-Jimoh E. Salami, "Parameter Estimation of Transient Mltiexponential Signals Using SVD-ARMA and Multiparameter Deconvolution Techniques", International Journal of Computer Theory and Engineering, vol 4, no. 5, 2012, pp. 751-757.

[6] J. Scott and A. Parker, "Modern Guide to Spectral Analysis with SPICE", IEEE Circuits and Devices Magazine, vol. 11, no. 5, pp.10-16, September 1995. 